\title{
Validation of dose measurements by scintillating fiber optic dosimeters for medical applications
}

\author{
A. Correia ${ }^{1} *$, R. Pirraco ${ }^{2}$, C. C. Rosa ${ }^{1,3}$ \\ ${ }^{1}$ INESC Porto, Optoelectronics and Electronics Systems Unit, Porto, Portugal \\ ${ }^{2}$ Medical Physics Department, IPOFG Porto, EPE, Portugal \\ ${ }^{3}$ Department of Physics and Astronomy, Faculty of Sciences, University of Porto, Portugal
}

\begin{abstract}
Organic scintillators have been promoted and widely used in scintillating fiber-optic dosimeters (SFOD) due to their tissue-equivalent characteristics, small sensitive volume combined with high spatial resolution, and emission of visible light proportional to the absorbed electron and gamma dose rate. In this paper we will present the validation of Monte Carlo simulations of dose measurements assisted by scintillating fiber optic dosimeters operating in the visible spectral range, in the context of the development of fiber optic dosimeters targeted to Brachytherapy. The Monte Carlo simulation results are compared to measurements performed with SFOD test probes, assembled with BCF-60 (Saint Gobain) samples of $1 \mathrm{~mm}$ diameter and 0.35 to $1.5 \mathrm{~cm}$ length, coupled to PMMA optical fiber. The optical signal resulting from scintillation and Cherenkov light is transmitted through an additional optical fiber link to a remote measuring device. For SFOD probes irradiation a dedicated PMMA phantom was used. The results were validated against measurements obtained with a properly calibrated pinpoint ionization chamber (PTW). The probes were positioned in a radial arrangement, with a radioactive source at its center point. The $\gamma$-rays source is a Nucletron Microselectron-V2 ${ }^{192} \mathrm{Ir}$. The dose curves are obtained according to the different positions in the phantom with the SFOD dosimeters. The system is able to use a Fiber Optic Multiplexer (FOM) controlled with Labview software.
\end{abstract}

Keywords: Dosimetry, scintillating fibers, brachytherapy.

\section{INTRODUCTION}

An effective brachytherapy treatment depends on the accuracy of radiation delivery to the target volume, while limiting the dose delivered to surrounding healthy organs and tissues. The effectiveness can be assessed by the use of proper dose measurement devices, preferably in situ and in real time, providing a delivered dose history record for exposed body volumes. According to the IAEA organization [1], errors in the specification of the source activity, dose calculation or in the quantities and units resulted in incorrect prescribed doses. Accidents are caused mainly by human mistakes, badly implanted sources or dislodged sources and equipment failure, where a lethal dose was delivered to the patients. For some brachytherapy sources, vendors assign large uncertainties to their stated calibration values, in some cases up to $\pm 10 \%$ [1]. So the need for real-time measurements is becoming more important and researchers are continually looking for ways to improve dosimetric systems, increasing the sensitivity and reducing costs [2] and design the probes with an optimized housing, transducer stability and biocompatibility [3, 4].

Proved to be essential in several application fields, such as cardiovascular, oncology and dermatology, among others, the optical fibres sensors must be capable to operate as stand-alone devices, must provide low signal attenuation, and avoid handling problems, but also ensuring safe operation and working conditions if in ionizing radiation environments [5,6].

Highlighted as an interesting alternative for the measurement of the absorbed dose, scintillators have been promoted by their higher energy resolution, excellent linearity in high temperature environments, ultrafast rise times, adequate mass absorption coefficients and tissue-equivalent characteristics [7-9]. The scintillating response of these materials is excited by ionizing radiation, where some part of the absorbed energy is re-emitted as light in the UV-VIS wavelength range, (and most commonly up to $550 \mathrm{~nm}$ ). This process is also found in applications such as gamma cameras in medical diagnostics, high energy particle physics experiments, x-ray security, computed tomography and radiation detectors [3, $8]$.

*Corresponding author: amrc@inescporto.pt 
Nowadays there are already fiber optic dosimeters intended to be used for in vivo real-time dosimetry in brachytherapy [9], but there is room for optimization and sensitivity enhancement. The most common problems on SFOD development is sensitivity, measured dose linearity, fibers fluorescence and Cherenkov effect radiation, the latter being responsible for a general loss of sensitivity and performance, and commonly referred to as stem effect [8]. The radiation from Iridium pellets $\left({ }^{192} \mathrm{Ir}\right)$, an isotope commonly used in brachytherapy is able to produce secondary electrons in irradiated materials with sufficiently high energies for Cherenkov radiation production in optical fiber, within a peak spectral range between 400 to $480 \mathrm{~nm}$. Since the Cherenkov radiation is proportional to $1 / \lambda^{3}$, where $\lambda$ is the wavelength of the Cherenkov light, the use of long wavelength scintillators is a method of avoiding the influence of stem effect on the detected signal [10].

We have been developing a SFOD multi-sensor, targeted to dose measurements in radiotherapy environments, exploring the use of alternative scintillators with emission bands in the NIR range of light spectrum. The setup uses alternative detectors, aiming at a lower prototype cost. Due to the specificity of the configuration and the conditional access to the brachytherapy facilities, a Monte Carlo simulation has been implemented to allow the simulation of most conditions and reduce the dependence of real life measurements. To be able to use these simulations, the code needs to be validated with real measurements taken with well-known experimental conditions. The work described in this paper presents the validation of the Monte Carlo simulation results, against the measurement performed with a calibrated Ionizing Chamber (the reference detector), and measurements of radioluminescent signal acquired with different light detectors. The linearity of these detectors is also established, by comparison with the results of the reference detector.

\section{MATERIALS AND METHODS}

\subsection{Radiation Source}

Measurements were performed at the Brachytherapy facilities of the Instituto Português de Oncologia Francisco Gentil (IPO-Porto), at Porto, Portugal. The $\gamma$-rays source is a Nucletron Microselectron-V2 with Iridium ${ }^{192}$ Ir pellets, encapsulated in a thin stainless steel capsule ( $1 \mathrm{~mm}$ diameter by $3.5 \mathrm{~mm}$ length cylinders) with an activity of 3.64Ci. The positioning and displacement of the ${ }^{192} \mathrm{Ir}$ source are controlled with a remote afterload. For the Monte Carlo numerical simulations, the energy spectrum was obtained from Cho et al. [11]. The geometry of the seed implementation is described by Chiquita [12].

\subsection{Scintillating Fiber-Optic Dosimeters (SFODs)}

The SFODs probes are made of a radioluminescent material (BCF-60, Saint-Gobain) with lengths between $3.5 \mathrm{~mm}$ to $15 \mathrm{~mm}$ coupled to a PMMA (poly-methyl-methacrylate, $\mathrm{C}_{5} \mathrm{H}_{8} \mathrm{O}_{2}$ ) fiber (RS Electronics) with $1000 \mu \mathrm{m}$ diameter and two meters long, which collects and transports radiation to a remote light measuring device, as illustrated in figure 1. The commercial organic scintillator BCF-60 is made out of a polystyrene-based core and a PMMA cladding and with an extra mural absorber often used to eliminate optical crosstalk and radiation-resistance characteristics. The scintillation yield is proportional to the dose-rate, emitting approximately 7100 photon/MeV, hence it is suitable for dosimetry, and is characterized by an emission peak at $530 \mathrm{~nm}[13]$.

The SFODs materials allow cheaper, thin, flexible, robust and lightweight dose transducers, also being immune to electromagnetic and chemical interferences, and most notably are closer to tissue-equivalent radiation behaviour than silica fibers, essential for accurate dose measurements without complex calibration processes [8, 14].

\subsection{Phantom and Ionization Chamber for dose measurements}

For ${ }^{192} \mathrm{Ir}$ and other high-energy sources, absorbed-dose water equivalence is less dependent on phantom composition, so that commercial plastics such as PMMA can be used with lower correction uncertainties due to knowledge of their composition [15]. As presented in figure 1b, the cylindrical PMMA phantom, with $20 \mathrm{~cm}$ of diameter and $12 \mathrm{~cm}$ of height, contains, five holes spaced from the center of the phantom central axis at different radial position (namely at 10 , $15,20,30$ and $40 \mathrm{~mm}$ ) from the Iridium pellet, and is fully described in ref. [12].

For validating the point-dose measurements, a cylindrical ionization chamber (PinPoint/PTW - ref. 31016 , with $16 \mathrm{~mm}^{3}$ of detection volume) built from water-equivalent materials is suggested to minimize detector over-response to variations in the photon spectrum as a function of measurement position, with an excellent stability, linear response to absorbed dose, small directional dependence, beam-quality response independence, and traceability to a primary calibration standard to avoid dose measurement errors $[16,17]$. The ionization chamber connected to a PTW Unidos electrometer has been inserted into the PMMA phantom at different radial positions for probe validation and linearity assessment. To 
minimize the contribution of scattered radiation, the source and the chamber was placed in the center of the room and well above the floor, at least $1 \mathrm{~m}$ from any wall or floor [1].
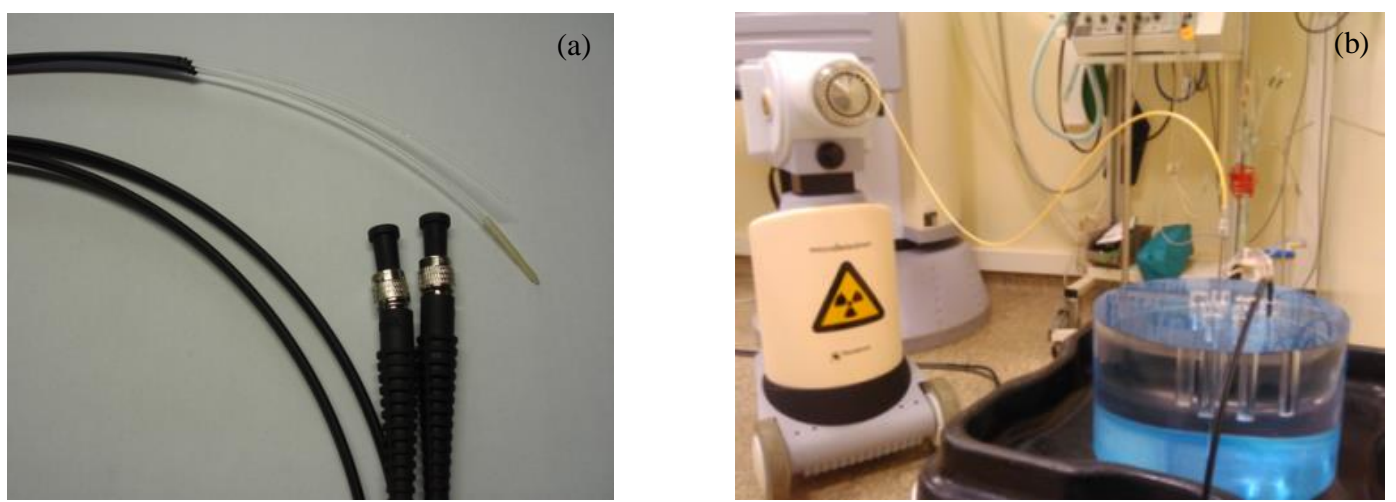

Figure 1. a) Twin fiber cable with scintillating material (BCF-60) and without (reference for stem effect measurement) and b) phantom used in dose measurements at IPO-Porto.

\subsection{Monte Carlo simulations}

The dose was estimated from Monte Carlo simulations, considering the same experimental geometry and conditions. This computational dosimetry technique plays an important role in establishing what is termed "reference-quality" dose distributions used to implement the TG-43 dose calculation formalism [1]. The Monte-Carlo models predict dose per unit contained activity, where a history represents the interactions undergone by a single emitted photon or electron, which can be related to contained activity by disintegration probabilities and branching ratios for complicated decay structures.

\subsection{Schematic diagram of the developed prototype}

The SFOD probes are coupled to an optical fiber that collects and transports radiation to a remote light measuring device. A sample of whole bare fiber was also prepared for stem effect estimation, placed in the water-phantom holes at $69 \mathrm{~mm}$ depth in order to suppress the Cherenkov light background.

The SMA terminated SFOD probes were linked to another low OH multimode silica fiber (Thorlabs) with $1000 \mu \mathrm{m}$ core diameter, and $10 \mathrm{~m}$ length, either directly through an SMA-SMA connector, or interfaced with a Fiber Optic Multiplexer ( $8 \times 2$ channels, Avantes). To maximize the optical coupling of the radioluminescent signal to the transmitting optical fiber an index matching gel (Thorlabs) was used. The radioluminescent signal was detected in the visible (320-1100 nm) spectral window (Fig. 2), with different detectors (PDF10A from Thorlabs and MPPC-128 from Hamamatsu). The femtowatt photo-receiver combines a selected ultra-low noise Si photodiode with a specially designed trans impedance amplifier offering extremely high gain. The MPPC (multi-pixel photon counter) contains a thermoelectrically cooled device capable of detecting ultra-low light, in the visible spectral windows typical between 320 to $900 \mathrm{~nm}$. The electrical signal was acquired with a NI DAQ USB-6351, and the control and signal processing were performed with the aid of Labview software (National Instruments).

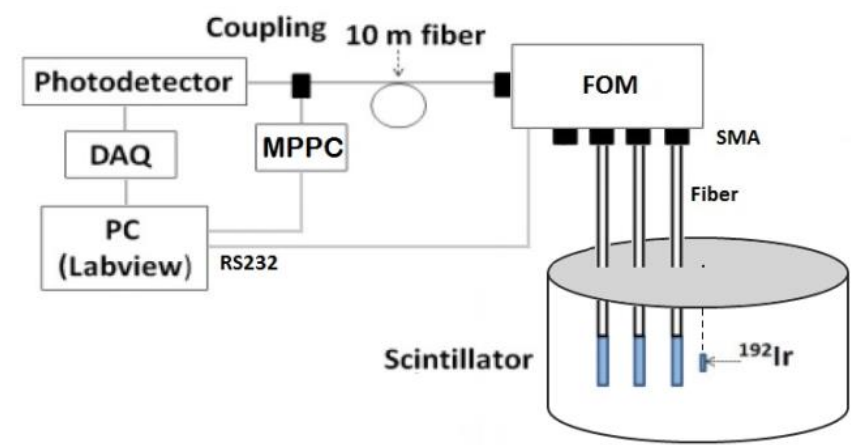

Figure 2. Schematic diagram of the developed prototype placed within a PMMA phantom, in order to make feasible irradiation of radioluminescent samples with ${ }^{192} \mathrm{Ir}$. 


\section{EXPERIMENTAL RESULTS}

\subsection{Source position}

According to the geometry of figure 2 and from the geometrical parameters of source and dosimeter, one can note that the seed is generally smaller than the scintillating material at the fiber tip, and that the measurement should be highly sensitive to the relative alignment of the Iridium source and the radiation detector (either the SFOD or the ionization chamber - IC). Hence, the first experimental step addresses the determination of the correct seed position corresponding to the minimum distance between source and dosimeter, and being this geometry the one corresponding to the Monte Carlo simulations. Also, when the source is positioned at this point, we expect the IC current to be maximized and the uncertainty in the reference air kerma rate determination, due to positional uncertainty, to be minimized [1].

Figure 3a shows the acquired SFOD signal when varying the source position along a vertical axis, coincident with the center of the PMMA phantom, and parallel to the $1 \mathrm{~cm}$ away SFOD axis. The SFOD contained a $15 \mathrm{~mm}$ long, and $1 \mathrm{~mm}$ diameter scintillating tip (BCF-60). The phantom was positioned at the centre of the Brachytherapy room (IPO-Porto). The chosen irradiation time was $5 \mathrm{~s}$. The maximum response is retrieved from the graph and used for posterior measurements with the dosimeter on that same phantom. This procedure needs to be performed for every single SFOD probe and for the PTW/PinPoint chamber.

Figure $3 \mathrm{~b}$ presents the radioluminescent signal (RL) detected by the $15 \mathrm{~mm}$ SFOD at different radial positions (SFOD seed separation), and collected after the fiber link by a MPPC detector. The detected radioluminescent signal shows an abrupt rise as soon as irradiation is switched-on, i.e the seed is quickly guided and positioned inside the phantom at the optimum position, and decaying behaviour when irradiation is turned-off, i.e the seed recoiled to the Nucletron radiation protected housing. The main problem in the design of a scintillator dosimeter is the background signal, so to isolate the Cherenkov contribution a simplest and most reliable method were adopted, using a twin detector, identical to the first but without a scintillator material that collects only the Cherenkov contribution which can be subtracted.
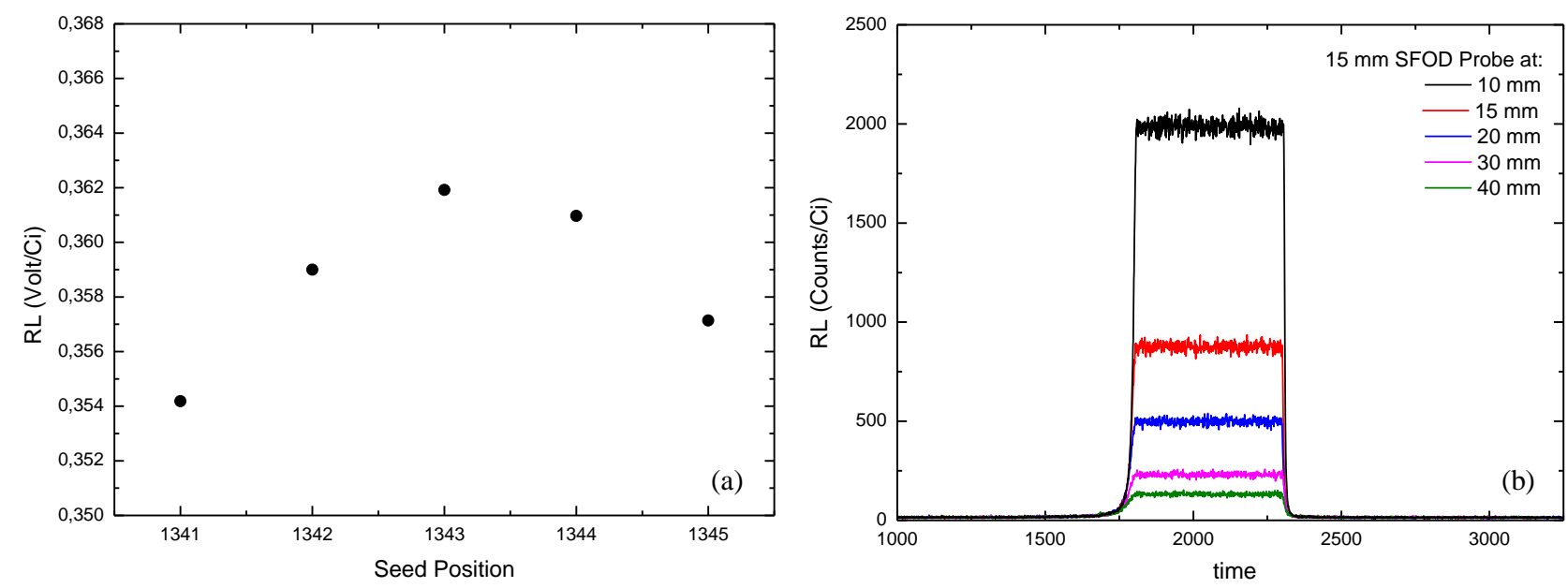

Figure 3. a) Calibration of the ${ }^{192}$ Ir position, reaches the maximum response for a SFOD with a scintillator of $15 \mathrm{~mm}$ length in the radial position $(10 \mathrm{~mm}$ ) obtained with a PDF10A detector and b) Response obtained from the MPPC (10ms integration time; threshold of 1.5 p.e. during an irradiation time of $5 \mathrm{~s}$ to a $15 \mathrm{~mm}$ SFOD at different radial positions.

\subsection{SFOD dose measurements}

The measurements obtained with three SFODs with 3 different BCF60 sample lengths ( $3.5 \mathrm{~mm}, 5 \mathrm{~mm}$ and $15 \mathrm{~mm}$ ) and $1 \mathrm{~mm}$ diameter of are shown in figure 4. In order to maximize signal to noise ratio, the MPPC photo-detector was used. The radial dose measurements were fitted to allometric functions $D(r)=a r^{b}$ with the purpose of comparing response behaviour along radial position for the different measuring conditions. Data is shown in figure 4, with the fitting functions identified in the legend. A good agreement with the chosen fit function behaviour is observed. 


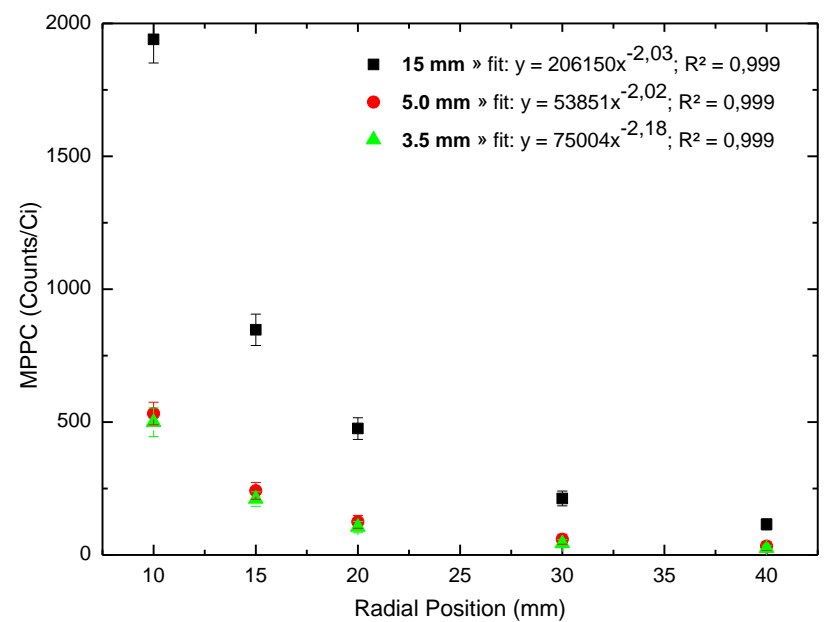

Figure 4. SFODs response in function of phantom radial positions and its fits equations (with background signal removed), measured by the MPPC (int. time: $10 \mathrm{~ms} ; 1.5$ p.e.).

As expected, a larger (longer) scintillator results in a higher scintillation yield, and the signal scales with the scintillating volume. The allometric parameter is close to $b=-2$. That is also in accordance with our expectations, since the SFOD extensions are equal or larger than the seed one, and the SFOD detection volumes, although small (2.74, 3.92 and $11.78 \mathrm{~mm}^{3}$ ), cannot be considered to be equivalent to point detection conditions.

\subsection{Reference dose measurement with Ionization Chamber}

The validation reference dose was measured with the calibrated ionization chamber. We used a PinPoint chamber from PTW (Model 31016). Special care was taken regarding temperature equilibrium of both chamber and PMMA phantom. The PinPoint chamber has a small diameter cavity, with active volume of $16 \mathrm{~mm}^{3}$, and is sensitive to radial positional changes. The measured data is shown in figure $5 \mathrm{a}$.

\subsection{Monte Carlo dose estimation}

The MCNP5 code developed by the Los Alamos National Laboratory (USA) was used. The implemented simulation estimated the dose at the corresponding positions occupied by the SFODs and ionization chamber in the phantom. The obtained values are also shown in figure 5a. Data for both Ionization Chamber and Monte Carlo simulation were normalized to the values obtained at radius of $30 \mathrm{~mm}$ from the seed, where one expects the dose not to be as much dependent on the seed geometry, as at closer distances.
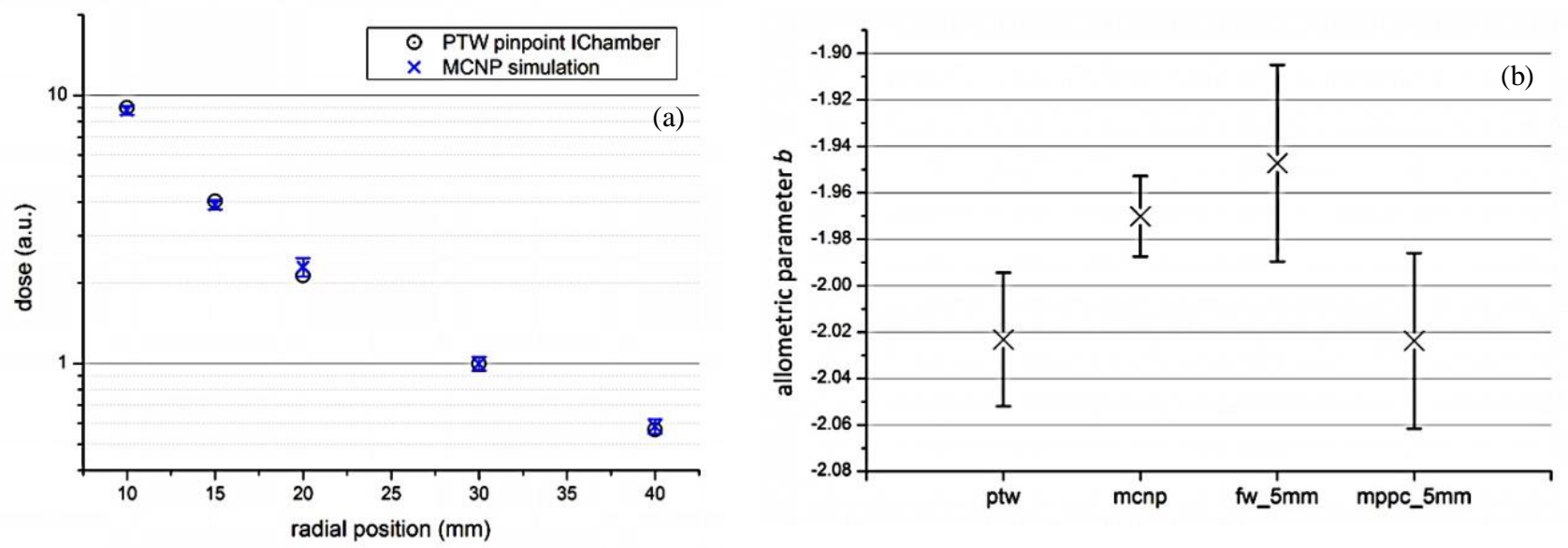

Figure 5. a) PTW PinPoint chamber dose measurements and simulation results and b) Comparison of spatial response from different dosimeters through the allometric $b$ parameter.

The Monte Carlo estimates have an accuracy error of less than $2 \%$ for larger radius, and less than $1 \%$ at closer distances. Nevertheless, the error bar in the graph is larger than that, varying from 4 to $6 \%$. The larger error arises from an estimate 
of dose variation at the detector, as a consequence of misalignment of seed and dosimeter, within a range of -4 to $4 \mathrm{~mm}$. We don't expect such large misalignments (see section 3.1), but whenever the dosimeter is replaced some variation might occur and should be compensated by seed position determination. This is a scenario only viable during the test phase of the SFOD.

In figure $5 \mathrm{~b}$, the allometric parameter $b$ obtained from the data corresponding to photo-detector and MPPC detection paired with the SFOD of $5 \mathrm{~mm}$ length, is compared with the ones obtained from reference data and Monte Carlo simulations.

\section{CONCLUSION}

This work presents SFODs probes employed in a single experimental set-up to evaluate the dose distribution of the microSelection ${ }^{192}$ Ir source in different location, which can be a promising candidate of in vivo and on-line dose monitor in the brachytherapy environments. With this setup, we have validated a Monte Carlo simulation that will allow us in the future to have a monitoring tool to assess the performance of SFOD dosimeters developed in the group. The measurements were performed with a cylindrical PMMA phantom and the results compared by simply fitting the data to an allometric curve. It should be noted that for a point radiation source we would expect the $b$ parameter to be around 2 , since the emission would be expected to be isotropic and follow a decay with the inverse of the square of the distance source-detector. Closer to the seed there may be a deviation from this law, since the seed as an elongated shape, deviating from the ideal point source response, and thus creating a different radial scaling for small separation distances and different alignment angles. Also, the measurements and quality of allometric fit will be affected by the physical dimensions and geometry of the sensing device. Still, the presented results are meaningful, and close to what was expected. The dose measurements were carried by low cost detectors and a Multi-pixel photon counter (MPPC) and with different BCF-60 probe lengths, and with an ionization chamber. The results are in agreement with each other and also comparable to both experimental and Monte Carlo estimates.

\section{ACKNOWLEDGEMENTS}

This work is financed by the ERDF - European Regional Development Fund, through the COMPETE Programme, ref. FCOMP-01-0124-FEDER-010960, and by national funds through FCT - Fundação para a Ciência e Tecnologia, project FIBDOSE-PTDC/SAU-BEB/108644/2008. The authors also acknowledge the grant from the host institution, INESCPorto, ref:559/BI_B1D/11, and the support given by the Brachytherapy Service at IPO-Porto.

\section{REFERENCES}

[1] IAEA, "Calibration of Brachytherapy Source, Guidelines on Standardized Procedures for the Calibration of Brachytherapy Sources at SSDLs and Hospitals," International Atomic Energy Agency, IAEA-TECDOC-1079, (1999).

[2] O'Keeffe, S., Fitzpatrick, C., Lewis, E. and Al-Shamma'a, A.I., "A review of optical fibre radiation dosimeters," Sensor Review 28, 136-142 (2008).

[3] Jang, K., Cho, D., Yoo, W., Park, B., Moon, J., Kim, S., Cho, Y., “A Scintillating Fiber-optic Dosimeter for Co60 Radiotherapy," IEEE 978(1), 4244-5335 (2009).

[4] Therriault-Proulx, F., et al., "HDR in vivo dosimetry using a PSD," Medical Physics 38(5), (2011).

[5] Patel, NP., et al., "In-air calibration of an HDR ${ }^{192}$ Ir brachytherapy source using therapy ion chambers," J. Cancer Res. Ther. 1(4), (2005).

[6] Sporea, D., Sporea, A., O’Keeffe, S., McCarthy, D. and Lewis, E.,’Optical Fibers and Optical Fiber Sensors Used in Radiation Monitoring," Select Topics on Optical Fiber Technology, ISBN: 978-953-51-0091-1 (2012).

[7] Frelin, A-M., Fontbonne, J-M., Ban, G., Colin, J. and Labalme, M., "Spectral Discrimination of Cerenkov Radiation in Scintillating Dosimeters," Med. Phys. 32, 3000-3006 (2005).

[8] Suchowerska, N. et al., "A fibre optic dosimeter customised for brachytherapy," Radiation Measurements 42, 929-932 (2007). 
[9] Andersen, C. E., et al., "Characterization of a fiber-coupled Al2O3:C luminescence dosimetry system for online in vivo dose verification during Ir-192 brachytherapy," Medical physics 36(3), 708 (2009).

[10] Law, S. H., Suchowerska, N., McKenzie, D. R., Fleming, S. C. and Lin, T., "Transmission of Cerenkov radiation in optical fibers," Opt. Lett. 32, 1205-1207 (2007).

[11] Cho, S. H., Muller-Runkel, R., and Hanson, W. F., "Determination of the tissue attenuation factor along two major axes of a high dose rate (hdr) ir source," Medical physics 26, 1492 (1999).

[12] Chiquita, S., "Dosímetro radioluminescente em fibra ótica para braquiterapia e radioterapia externa," MSc Thesis in Medical Physics, Faculdade de Ciências, Universidade do Porto (2011).

[13] Santiago, M., Prokic, M., Molina, P., Marcazzó, J., and Caselli, E., “A tissue-equivalent radioluminescent fiber optic probe for in-vivo dosimetry based on Mn-doped lithium tetraborate," IFMBE 25, 367-370 (2009).

[14] Shikama, T., et al., "Optical dosimetry for ionizing radiation fields by infrared radioluminescence," Measurement Science and Technology 17, 1103 (2006).

[15] Perez-Calatayud, J., Ballester, F., Das, R. K., DeWerd, L. A., Ibbott, G. S., Meigooni, A. S., Ouhib, Z., Rivard, M. J., Sloboda, R. S., and Williamson, J. F., "Dose calculation for photon-emitting brachytherapy sources with average energy higher than $50 \mathrm{keV}$, , Med. Phys. 39, 5 (2012).

[16] Low, D. A., Moran, J. M., Dempsey, J. F., Dong, L., and Oldham, M., "Dosimetry tools and techniques for IMRT," Med. Phys. 38, 3 (2011).

[17] Kim, K. A., Yoo, W. J., Jang, K. W., Moon, J., Han, K. T., Jeon, D., Park, J. Y., Cha, E. J., and Lee, B., "Development of a fibre-optic dosemeter to measure the skin dose and percentage depth dose in the build-up region of therapeutic photon beams," Radiation Protection Dosimetry 153(3), 294-299 (2013). 